\title{
LOS PUEBLOS FERROVIARIOS DE LA PAMPA Un proyecto a dos escalas
}

\author{
Dra Arq. Mónica Martínez \\ Universidad Nacional de Córdoba. Facultad de Arquitectura, Urbanismo y Diseño. \\ arqmo.martinez2016@gmail.com
}

\section{RESUMEN}

Esta presentación forma parte del trabajo de investigación Los pueblos del desierto: el procesos de ocupación y urbanización del Territorio Nacional de La Pampa, Argentina (Martínez, 2016). Este territorio ocupa la posición central de Argentina y contiene la faja occidental de la llanura pampeana, el sector de mayor productividad agrícola y ganadera del país, de América Latina y en posición privilegiada en el mundo. A fines del siglo XIX, con el desarrollo industrial y la demanda de materias primas de los países desarrollados, el entonces Territorio Nacional de La Pampa entra en un proceso de acelerada de ocupación y urbanización, transformando radicalmente un amplio espacio virgen en zonas productivas, e integrándolo rápidamente a la estructura nacional e internacional. Todos los acontecimientos que entonces se suceden, marcan una historia desconocida de este territorio que -en parte- se narra en esta presentación: el trazado rural, la puesta en producción, el trazado ferroviario y el trazado de los pueblos. Estos aspectos denotan definitivamente los rasgos principales de la identidad de este territorio, otorgándole particularidades, que persisten y determinan el futuro de la actual provincia de La Pampa.

Palabras clave: territorio, pueblos, identidad, La Pampa.

\section{ABSTRACT}

This presentation is part of the research work The peoples of the desert: the processes of occupation and urbanization of the National Territory of La Pampa, Argentina (Martínez, 2016). This territory occupies the central position of Argentina, and contains the western belt of the Pampas plain, the sector of greater agricultural and livestock productivity of the country of Latin America and in a privileged position in the world. At the end of the 19th century, with the industrial development and the demand for raw materials from developed countries, the National Territory of La Pampa entered an accelerated process of occupation and urbanization, radically transforming a vast virgin territory into productive zones, and rapidly integrating it into the national and international structure. All the events that happened then mark an unknown history of the territory, and which is partly narrated in this presentation: the rural layout, the putting into production, the railway layout and the layout of the villages. These aspects definitively denote the main features of the identity of this territory, granting particularities that persist and determine the future of the present province of La Pampa.

Key words: territory, towns, identity, La Pampa 


\section{INTRODUCCION}

A mediados del siglo XIX, tras la unificación del gobierno nacional, las ideas de progreso y los modos para obtenerlo, homogenizan a la elite dominante en el país. Se desarrolla un consenso liberal que expresa el interés de los grupos propietarios, por dinamizar la economía y mantener estrechos vínculos con el mundo político. Los principales pensamientos de la época estaban sostenidos por las ideas de barbarie vs civilización (Sarmiento,1845), liberalismo como doctrina económica dominante, colonización e inmigración (Avellaneda,1876). Se propone insertar nuestro país en la economía mundial mediante su rol exportador de materias primas agrícolas y ganaderas, anexar nuevas tierras a la región pampeana consolidar el territorio nacional para su puesta en producción, ampliar el negocio de la tierra con participación de grandes terratenientes y especuladores. ${ }^{1}$

Hasta bien avanzado el siglo XIX, mientras el resto del territorio de Argentina se organiza en provincias, el Territorio Nacional de La Pampa está ocupado por una población indígena de etnias dominantes -ranqueles y pampas- de características nómades. Tras la política de conquista militar de 1879 y exterminio indígena, se dispone la enajenación de la totalidad de las tierras a manos de inversores extranjeros y latifundistas de la campaña bonaerense (Térzaga, 2012).

En este escenario, en las últimas décadas del siglo XIX y comienzos del XX, la porción oriental de este territorio nacional, con la extensión del ferrocarril, se coloniza y urbaniza, situación que transforma radicalmente su condición de ocupación anterior y caracteriza el desarrollo de la provincia de La Pampa definitivamente.
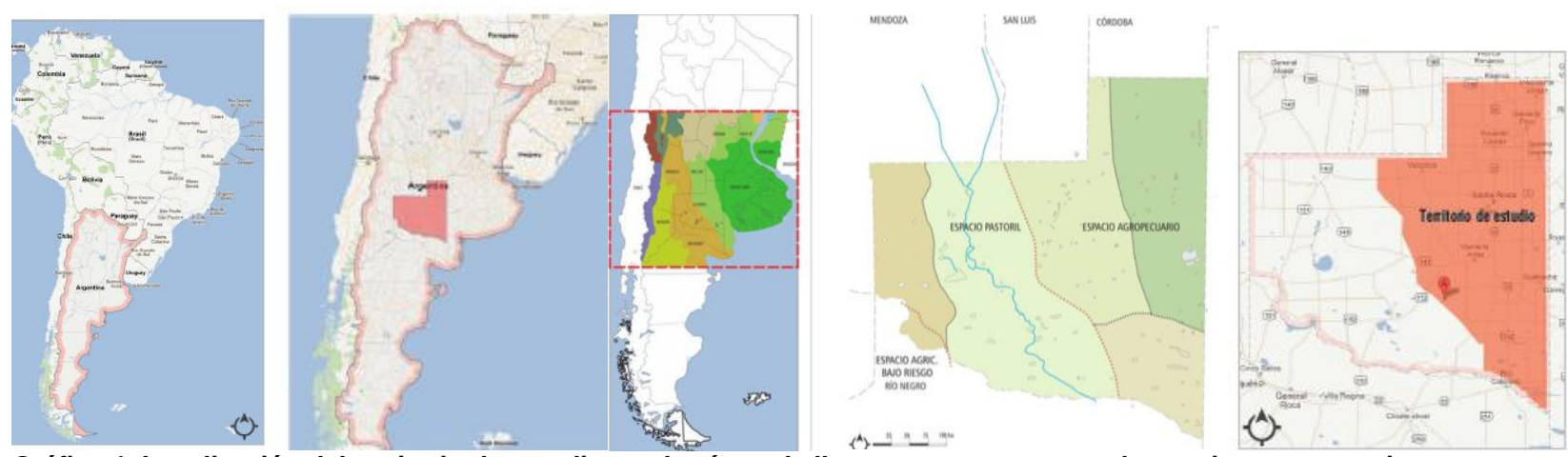

Gráfico 1. Localización del territorio de estudio en el país, en la llanura pampeana y en el espacio agropecuario.

Elaboración en base a fuente (LLup et al., 2008)

Sobre un paisaje de llanura cuasi absoluto, inmenso y vacío, marginal de toda civilización, una macro cuadrícula ordena y divide el territorio. Sobre ésta, el trazado ferroviario diferencia la ortogonalidad dominante de la grilla territorial; la disposición del cuadro de la estación y el edificio de la estación de pasajeros, define la localización del centro urbano, disponiendo en su entorno quintas y chacras de diferentes dimensiones. El análisis de la estructura funcional y la dinámica del proceso de ocupación de este territorio urbano-rural, se complementa con el análisis de su configuración morfológica, los elementos de la construcción del paisaje y los rasgos que lo identifican.

En esta presentación se plantea como objetivo central analizar morfológicamente la relación de los elementos que participan de la construcción del patrón dominante pueblos+ colonias+ ferrocarril e identificar la impronta de estos rasgos en la configuración paisajística de los pueblos de La Pampa. ${ }^{2}$ Para ello se analizan los elementos de la construcción del espacio en dos escalas: territorial y urbana.

Se propone explicar cómo los principales elementos de la estructura física y paisajística de estos pueblos están presentes en la escala regional y viceversa. A partir de la dialéctica entre estos aspectos, es posible

\footnotetext{
${ }^{1}$ Se entiende por región pampeana, la región amplia que comprende la provincia de Buenos Aires y parte de las provincias de Santa $\mathrm{Fe}$, Córdoba y La Pampa, con una gran aptitud para el desarrollo agrícola-ganadero.

${ }^{2}$ En adelante y en referencia al sector de estudio, se emplea el término territorio de La Pampa. Otros términos que se emplean para significar este espacio son: a) la pampa: en referencia a la región pampeana; b) Territorio Nacional de La Pampa o Pampa Central: es el nombre que adopta el territorio desde su conquista -a fines del siglo XIX-.
} 
inferir ciertos rasgos comunes, que constituyen las características principales de la identidad de los pueblos de La Pampa.

Para ello, el desarrollo se ordena en tres momentos. El primero refiere a La construcción de la identidad pampeana, donde se dispone el marco teórico de referencia y los antecedentes principales. Entre estos destacan los estudios de Jorge Ramos (1991) para la pampa bonaerense y de Joaquín Sabaté $(2004,2005)$ sobre la identidad del territorio. El segundo aborda los elementos de la construcción del territorio de La Pampa: el soporte natural, el trazado rural, el trazado ferroviario, los caminos rurales, las actividades productivas y las edificaciones, la estructura del territorio. El último comprende los elementos de la construcción de los pueblos ferroviarios de La Pampa: el cuadro de la estación, el trazado urbano, las calles principales, las plazas y las edificaciones, la estructura de los pueblos como síntesis.

En la elaboración de este desarrollo se recurre a fuentes primarias, planos de los centros urbanos y mapas del territorio en el momento de su fundación, Cartas del Instituto Geográfico Nacional (I.G.N.) y planos actuales de las localidades.

\section{LA CONSTRUCCIÓN DE LA IDENTIDAD PAMPEANA}

El caso del Territorio Nacional de La Pampa, representa un antecedente valioso en tanto la ocupación y colonización del territorio comienza conjuntamente con el trazado de los centros urbanos. Este hecho constituye una epopeya para el territorio argentino, consiguiendo en un plazo de escasas tres décadas, poner en producción y urbanizar cerca de $80.000 \mathrm{~km}^{2}$ de las tierras más productivas de la Argentina y el mundo.

El patrón dominante urbano-territorial que posibilita la transformación de un territorio inmenso y cuasi homogéneo contiene en su configuración morfológica y en el análisis de los elementos que la determinan, los rasgos principales de la identidad de La Pampa. Estos elementos han sido estudiados por Ramos (1991) para el territorio rural de la campaña bonaerense. ${ }^{3}$

Sin embargo, resulta importante acá, marcar la diferencia entre la pampa bonaerense -a la que refiere Ramosde la pampa que forma para de este estudio, donde el avance del capitalismo europeo hacia fines del siglo $\mathrm{XIX}$, no tuvo iguales consecuencias. Desde fines del siglo XVIII se desarrolla en la pampa bonaerense una cultura ganadera criolla, marcada por la fuerte presencia del gaucho pampeano. Un siglo después, La Pampa se incorpora al escenario productivo, extendiéndose naturalmente la cultura criolla de la pampa bonaerense, aunque más permeable a su transformación radical, dado la ocupación anterior indígena previa y su exterminio. Mientras en Buenos Aires sigue predominando la actividad económica ganadera, La Pampa, se muestra más abierta a la colonización agrícola del suelo.

Como fuera, gaucho e indio, no son bien vistos en la cultura dominante a fines del siglo XIX, y por ello necesitan ser civilizados. Estos hombres conforman un espacio de cualidad propia, como consecuencia de las interacciones que mantienen con el territorio que habitan y su medio ambiente. A los ojos de la gente urbanizada, el hábitat pampeano, es calificado como bárbaro, y necesita ser transformado. Exterminado el indio, La Pampa se presenta como territorio virgen, sin resistencia alguna. El patrón de asentamiento representado por las colonias, el ferrocarril, los pueblos, encarna el modelo de la civilización, poniendo a producir, definitivamente este suelo. De esta interacción, surge la construcción de la cultura física pampeana y de su identidad.

Los modelos de trazados de nueva fundación dominantes de fines del siglo XIX y principios del XX, se caracterizan por un cambio progresivo en la concepción del espacio rural, hacia su desarrollo agrícola, a través de su distribución en quintas, chacras; hasta su colonización, y desarrollo del tendido ferroviario. Los trazados de los pueblos y sus ejidos presentan características de los modelos republicano en base a la Ley de Colonización de 1876, el originado en el tendido ferroviario y el de colonización agrícola. El modelo republicano reemplaza el modelo colonial y establece una nueva relación entre el espacio rural y urbano. El ejido se constituye como la primera unidad territorial. Se propone un trazado en cuadrículas tanto en el núcleo urbano, como en el destinado a la producción. El modelo originado en la colonización agrícola de colonias, se caracteriza por una estrecha relación con el trazado rural, y por una ocupación de inmigrantes

\footnotetext{
3 Durante el período colonial, las ciudades desarrollan funciones administrativas respecto de un territorio definido en términos teóricos, desde donde se procede a la distribución de mercedes territoriales. Durante el período virreinal y comienzo del período independiente, se trazan pueblos como afirmación de las fronteras frente al avance indígena. En tanto, desde mediados del siglo XIX y comienzos del $X X$, los territorios que se anexan son colonizados y urbanizados, los centros urbanos se distribuyen de manera homogénea, desarrollando una fuerza centrípeta respecto de las colonias, como centros de servicios y concentración de la producción (Foglia, 1995).
} 
de diferentes grupos étnicos, dándoles esto características particulares, según su caracterización cultural. El modelo originado en el tendido ferroviario, fuertemente influenciado por la infraestructura ferroviaria.

La lectura de los rasgos de identidad del territorio según Ramos, se complementa con el aporte de experiencias en otros contextos de desarrollo. Sabaté (2004) estudia la identidad territorial y su aporte para proyectar el territorio. En su experiencia plantea algunos tópicos de análisis propositivos basados en el reconocimiento de los elementos patrimoniales -naturaleza y cultura- constituyentes de la estructura territorial; propone ciertos vínculos entre la forma del territorio y su transformación, y como éstos aportan pautas para la intervención del territorio.

La comprensión de las reglas de composición y formación inicial de los pueblos en el territorio de La Pampa, constituye un insumo para pensar y proyectar el espacio pampeano. Su conocimiento permite preservar el paisaje y su identidad, respetando su valor patrimonial, controlando los impactos negativos sobre la naturaleza y la cultura local.

\subsection{Los elementos de la construcción del territorio}

Los elementos que participan de la construcción del territorio de La Pampa, como componentes de su identidad, son el soporte natural, los trazados rurales, el trazado ferroviario, las actividades productivas y sus edificaciones, los pueblos.

Un análisis profundo de la geografía física del soporte natural del territorio en estudio permite realizar una lectura sutil de los diferentes paisajes naturales de La Pampa, contra la tendencia de la generalización del dominio de la planicie, la llanura, el horizonte (Caviglia et al., 2010).

En la porción de este territorio donde se asientan la mayoría de los pueblos de La Pampa, es posible reconocer diferentes paisajes y aptitudes de las tierras para su producción (Cano, 2004). El inventario integrado de los recursos naturales de la Provincia de La Pampa, reconoce en el territorio de estudio tres regiones y seis subregiones con características de clima, paisaje, vegetación.

Distintas conformaciones morfológicas de relieve (planicie, colina, loma, valle), suelo (médanos, tosca), clima (humedad, amplitud térmica, vientos dominantes), vegetación (pastizales, matorrales, arbustales, áreas con bosque abierto o cerrado), agua (depresiones, lagunas, salitrales), dan por resultado diferentes conformaciones paisajísticas y aptitud productiva del suelo.

Estas particularidades otorgan sutiles diferencias a las distintas regiones analizadas, tanto por las actividades económicas dominantes -ganadería, agricultura, producción forestal- como por las unidades productivas requeridas para ellas, lo que determina el tamaño promedio de las parcelas rurales. La región de la llanura oriental es la de mayor aptitud para el desarrollo agrícola-ganadero, considerándola una extensión de la llanura húmeda bonaerense, aunque con menor registro de lluvias; en tanto la región de la pampa central y la región meridional o depresión del Sudeste lo hacen en forma decreciente.

El trazado rural del vasto territorio pampeano se lleva adelante mediante su cuadriculación, este sistema de mensura y registro aplicado exclusivamente al territorio que se anexa tras la conquista del desierto, tiene sus antecedentes en la división de suelo norteamericano con fines similares. La cuadriculación del territorio ordena y estructura el más importante proceso de ocupación y urbanización de alcance regional. ${ }^{4}$

A la llegada de las expediciones militares La `Pampa está trazada por una red de caminos o huellas indígenas. Su disposición en red irregular es estratégica tanto en las comunicaciones que facilita integrando la totalidad del territorio, como en el conocimiento de sus cualidades naturales, referenciando en su recorrido lagunas, pastizales, zonas de descanso, abrigo, alimentación, protección. Los caminos indígenas iniciales son sendas irregulares sobre el espacio de La Pampa. La ordenación en cuadricula de este territorio desconoce esta disposición inicial, homogenizando el espacio geográfico de La Pampa.

\footnotetext{
4 Los antecedentes de la aplicación del modelo territorial norteamericano en el caso argentino, se encuentran en los pensadores y políticos de mediados del siglo XIX,81 Sarmiento y Avellaneda, quienes introducen las ideas norteamericanas durante la formación del Estado argentino, constituyéndose en referente directo de la transferencia de la legislación norteamericana a Argentina La grilla territorial norteamericana juega un rol central en la estrategia del Estado por el avance y control de las nuevas tierras, con consecuencias directas sobre los aspectos económicos (venta de tierras fiscales), fiscales, socio político (ventajas a la elite terrateniente) y urbanísticos (la cuadrícula como base de la subdivisión del territorio productivo y del trazado de los pueblos)
} 

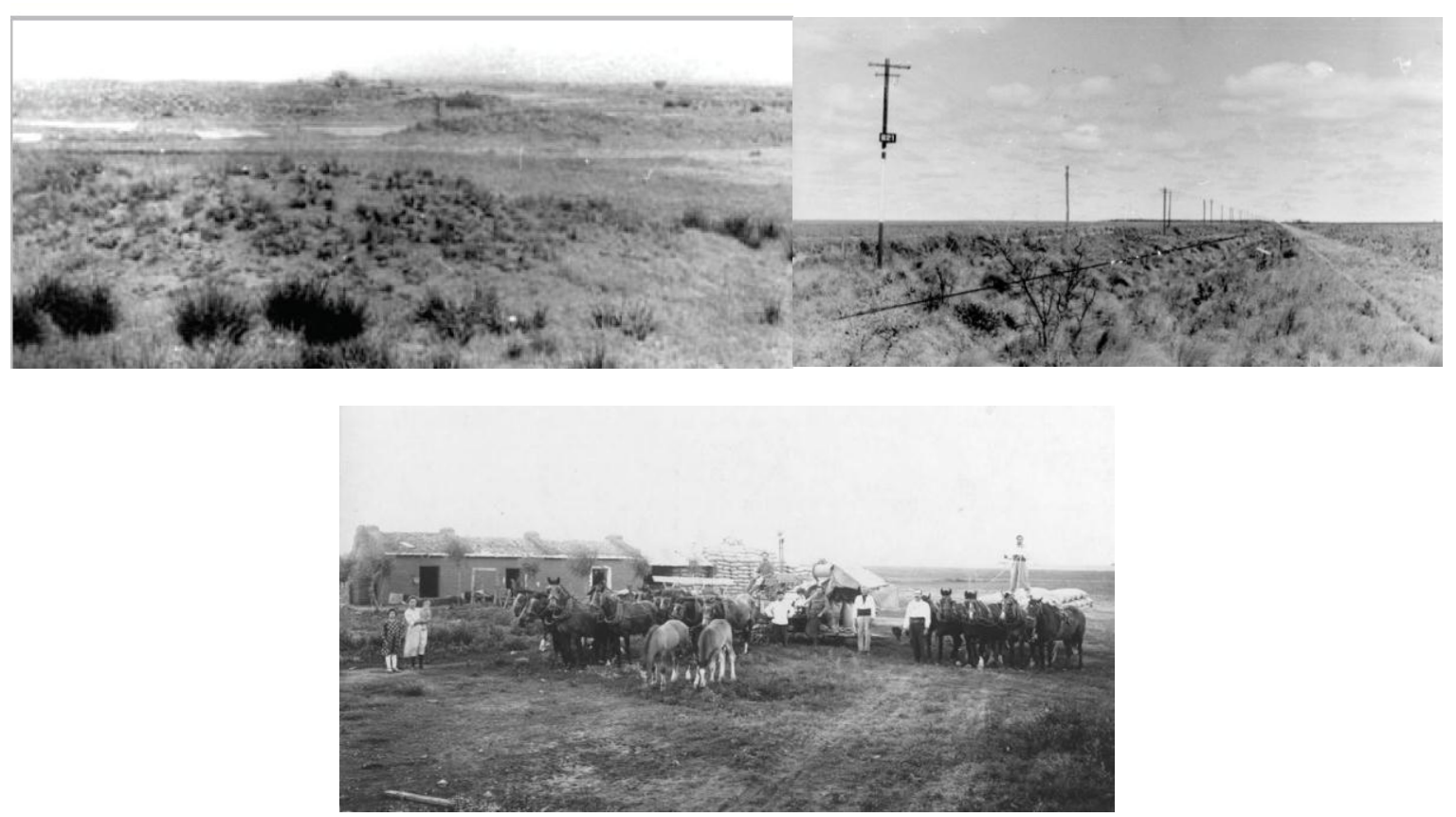

Gráfico 2. Fotos del paisaje dominante de llanura, el avance ferroviario y la producción rural de las colonias a fines del siglo XIX.

Fuente: Fototeca Bernando Graff, Archivo Histórico Provincial "Prof. Fernando E. Aráoz". Santa Rosa.

La Pampa. En https://fototecabernardograff.wordpress.com/.

Sobre este primer trazado, la pampa es centuriada y mensurada en grandes secciones 1.000 .000 de ha, fracciones y lotes de 10.000 ha. Las primeras trazas responden a grandes extensiones de tierra de 100.000 ha y más. Iniciado el siglo XX, sobre esta división, algunos lotes y fracciones incorporan un nuevo trazado, en secciones menores, para su puesta en producción agrícola, disponiendo colonias de 10.000 ha y chacras de 100 ha promedio. Las chacras reproducen internamente la ortogonalidad del trazado, ordenando los ingresos y la división de los campos con fines productivos agrícolas dominantes. ${ }^{5}$

La materialización efectiva de las parcelas rurales -sus límites- mediante el alambrado de los campos llevan a regularizar la disposición de los caminos, ordenándolos geométricamente, en densidad creciente a medida se reduce la unidad productiva. Los lotes que no se colonizan se mantienen en grandes superficies pudiendo desarrollar cascos de estancia. ${ }^{6}$
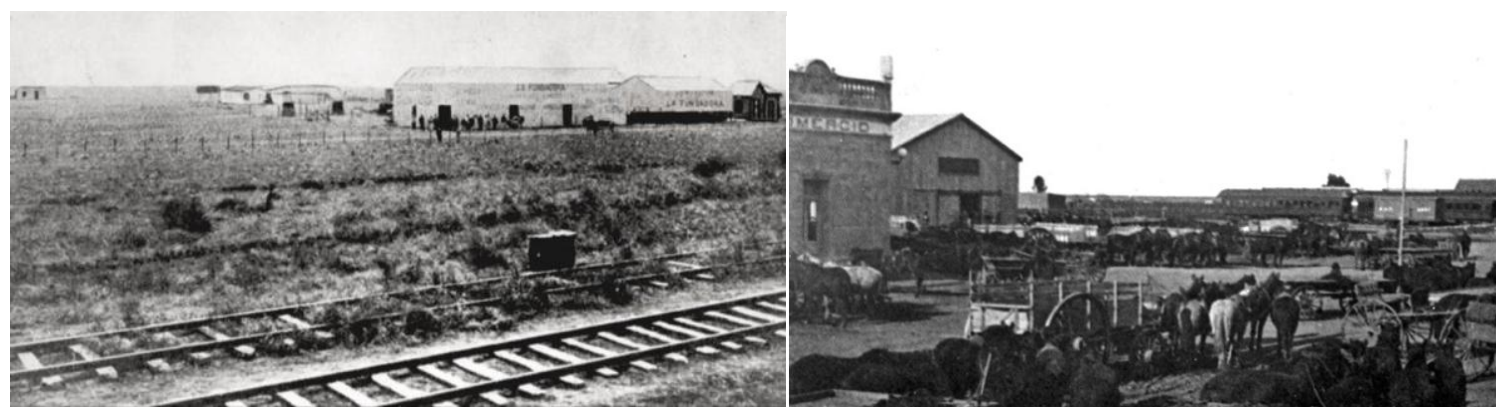

\footnotetext{
${ }^{5}$ Ley de frontera de 1878. En secciones de $100 \times 100 \mathrm{Km}$ (20 leguas kilométricas), numeradas de Norte a Sur y comenzando desde el Meridiano 5‥ La numeración se individualiza con números romanos: I, II, III, IV y más. Cada sección se divide en cuatro fracciones de $50 \times 50 \mathrm{Km}$, designadas A.B.C.D, en el sentido de las agujas del reloj, ubicando la A en el ángulo NO. Una fracción se subdivide en 25 lotes, unidad básica del parcelario, de $10 \mathrm{Km} \times 10 \mathrm{Km}(10.000 \mathrm{ha})$ numeradas de 1 a 25 .Un lote se divide en cuatro leguas de $5 \times 5 \mathrm{Km}$ (2.500 hectáreas) designadas con letras minúsculas a,b,c,d, ubicándose la a en el ángulo NO. El molino independiza de la lluvia y de los escasos cursos de agua.

${ }^{6}$ Se trata de implantaciones rurales con micro paisajes de matriz centro europea. En La Pampa, algunos de estos son cotos de caza de jabalí del monte pampeano.
} 


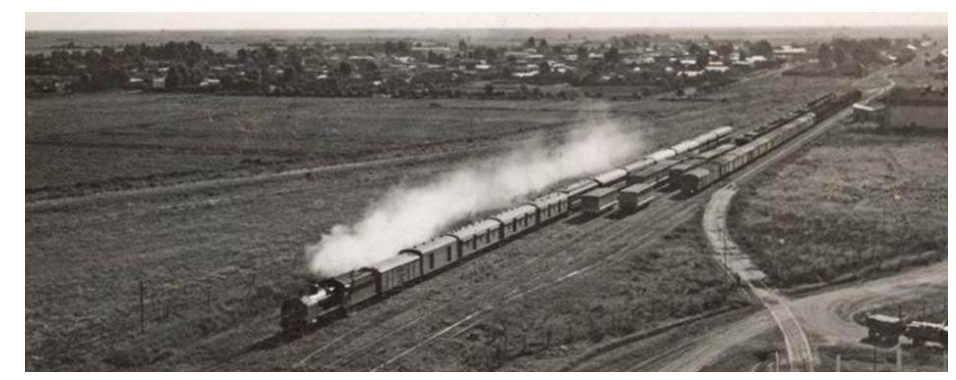

\section{Gráfico 3. Fotos del paisaje ferroviario, la dinámica entorno a las estaciones y el trazado de los pueblos a comienzos del siglo XX. \\ Fuente: Fototeca Bernando Graff, Archivo Histórico Provincial "Prof. Fernando E. Aráoz". Santa Rosa.} La Pampa. En https://fototecabernardograff.wordpress.com/.

La geometría de los trazados y la disposición racional, ordenada, uniforme y simétrica del suelo rural, caracteriza significativamente el paisaje pampeano. La condición de espacio plano dominante, la noción de escala y de límite -el espacio inconmensurable- constituye aspectos identificatorios del paisaje pampeano.

Desde la centuriación del territorio hasta el trazado urbano en cuadrícula, los elementos culturales que se incorporan, contribuyen a mensurar lo inmensurable, cualquier construcción singular pone el horizonte como fondo y referencia el tiempo y el espacio (Bulgheroni, 1994).

No todo lo que se cuadricula se coloniza. El trazado de las colonias sobre esta macro cuadrícula, y su prosperidad, depende de la subdivisión del suelo y su relación con la unidad productiva mínima por subregiones; la mayor o menor proximidad y dependencia con el trazado ferroviario y su conexión con los puertos, aspectos todos de la configuración física resultante que adopta el patrón urbano territorial dominante. ${ }^{7}$

El trazado ferroviario se dispone con una lógica diferente. Se trata de unir geográficamente dos puntos, y trazar el camino más corto, adaptando éste a las condicionantes naturales conforme pendientes, radios de curvaturas, desniveles. Está demostrado que el ferrocarril en La Pampa, se apoya en el conocimiento que desarrollan los indígenas sobre estas tierras. Los primeros trazados ferroviarios de La Pampa copian la dirección dominante de las principales rastrilladas, que también representan, las entradas del ejército de conquista (Mollo, 2009).

La dirección que las compañías ferroviarias siguen en el trazado del ferrocarril en La Pampa, atan los puertos de procedencia -Buenos Aires y Bahía Blanca- con los dos primeros centros trazados como colonias militares en La Pampa -Victorica y Genera Acha- desplegando una primera estrategia de conquista económica sobre el territorio. Mientras en la provincia de Buenos Aires, el sistema insular es dominante, ya que la mayor parte de los centros ya están trazados cuando llega el ferrocarril, en La Pampa, unos pocos centros se trazan previos a éste.

La cuadrícula se modifica con la incorporación del ferrocarril. La dirección que infringe éste al espacio monótono de la grilla regular inicial, genera un nuevo referente que condiciona totalmente el proceso urbanizador. El territorio se traza con las líneas de las distintas compañías que entran a La Pampa: Ferrocarril del Oeste, Ferrocarril Bahía Blanca al Noroeste y Ferrocarril del Sur. Cada 18 a $20 \mathrm{Km}$ se disponen los cuadros de estación. De las cerca de 100 estaciones que se trazan, 79 derivan en centros urbanos y/o rurales en su entorno. Paralelo a los casi $1500 \mathrm{Km}$ de vías se dispone el tendido telegráfico y los caminos rurales de servicio. ${ }^{8}$

\footnotetext{
${ }^{7}$ Las condiciones de gestión de las compañías de tierras o del propietario del suelo; los sistemas de arrendamiento y la facilidad otorgada a los colonos para su puesta en producción. La ocupación inmigrante que se incorpora a comienzos del siglo XX, es la base de la colonización y puesta en producción agrícola del territorio. Para 1920, de los 125.000 habitantes que La Pampa posee, el 28,3\% son migrantes transoceánicos.

${ }^{8}$ La primera compañía avanza desde el Sur, buscando conectar el puerto de Bahía Blanca con las nuevas zonas productoras. En 1891 , en plena crisis, ingresa a La Pampa la compañía de capitales ingleses Ferrocarril Bahía Blanca and North Western Company Limited (F.C.B.B.\&NO). La compañía F.C.B.B.\&NO proyecta atravesar el territorio de La Pampa, buscando conectar el puerto de Bahía Blanca con las provincias del Norte, Cuyo y Chile. El primer ramal de esta compañía entra desde Nueva Roma (Buenos Aires) hasta Toay (La Pampa), deteniéndose en el centro de La Pampa. Seis años después, una segunda compañía avanza hacia el Oeste, desde los límites de Buenos Aires con el entonces territorio nacional, conectando La Pampa con el puerto de Buenos Aires. En 1897, ingresa el Ferrocarril del Oeste de Buenos Aires (F.C.O). Busca atravesar el desierto "infinitamente" en dirección Oeste. El primer ramal entra a La Pampa
} 
En los límites de las distintas fracciones en que se divide el suelo se van disponiendo los caminos, cada distancias que varían según la división catastral en cuadrícula de los campos. A medida se acercan a los pueblos, la densidad de los caminos de la malla se incrementa. En los territorios colonizados, las chacras ordenan estos cada $1 \mathrm{~km}$ o $1 / 2 \mathrm{Km}$ de distancia.

Los polvorientos caminos rurales que van de los campos a la estación son, inicialmente, huellas recorridas por carros y carretas que siguiendo las líneas divisorias ortogonales -entre propiedades- llevan la producción de las colonias a los pueblos. Apenas atraviesan un caserío, arriban al cuadro de la estación; allí se encuentran con el camino de hierro que lleva la producción al puerto, cumpliendo con el mandato del modelo económico agroexportador.

En la zona de quintas y chacras, algunos de estos caminos, presentan conjuntamente con las líneas divisorias de propiedades, enfiladas de árboles de gran porte -eucaliptus australianos- que además de fijar los médanos, referencian el espacio y lo recalifican, construyendo los rasgos dominantes de la transformación del paisaje pampeano. La secuencia rítmica del trazado sobre el plano, toma la tercera dimensión, mediante filas de verde que acompañan por tramos las sendas, especialmente las que definen el acceso a las chacras para envolver luego la vivienda rural -en el centro- protegiéndola de los trabajosos vientos de La Pampa. Barreras verdes se anteponen al horizonte, contribuyen a parar el viento y generan sensación de protección, contención, límite. Siguiendo la lógica geométrica del trazado de los caminos y disposición de los alambrados, el verde no puede más que ordenarse ortogonalmente en este espacio (Bulgheroni, 1994).

El encuentro de dos caminos rurales ortogonales es el lugar propicio para la localización de las primeras actividades productivas y sus edificaciones, los almacenes, puntos estratégicos de parada, abastecimiento, descanso en las travesías por La Pampa. Estas ocupan un lugar importante en la red de caminos rurales, actúan como postas de las mensajerías. Algunas, referencian físicamente, el lugar de fundación de los primeros pueblos y/o trazado de los cuadros ferroviarios. Hasta los primeros centros urbanos, estos representan puntos de reunión en el medio de la pampa, condensadores sociales en el desierto, centros de abastecimiento y despacho de bebidas, albergue, que aceptan la modalidad del trueque (Pérez Amuchástegui, 1969).
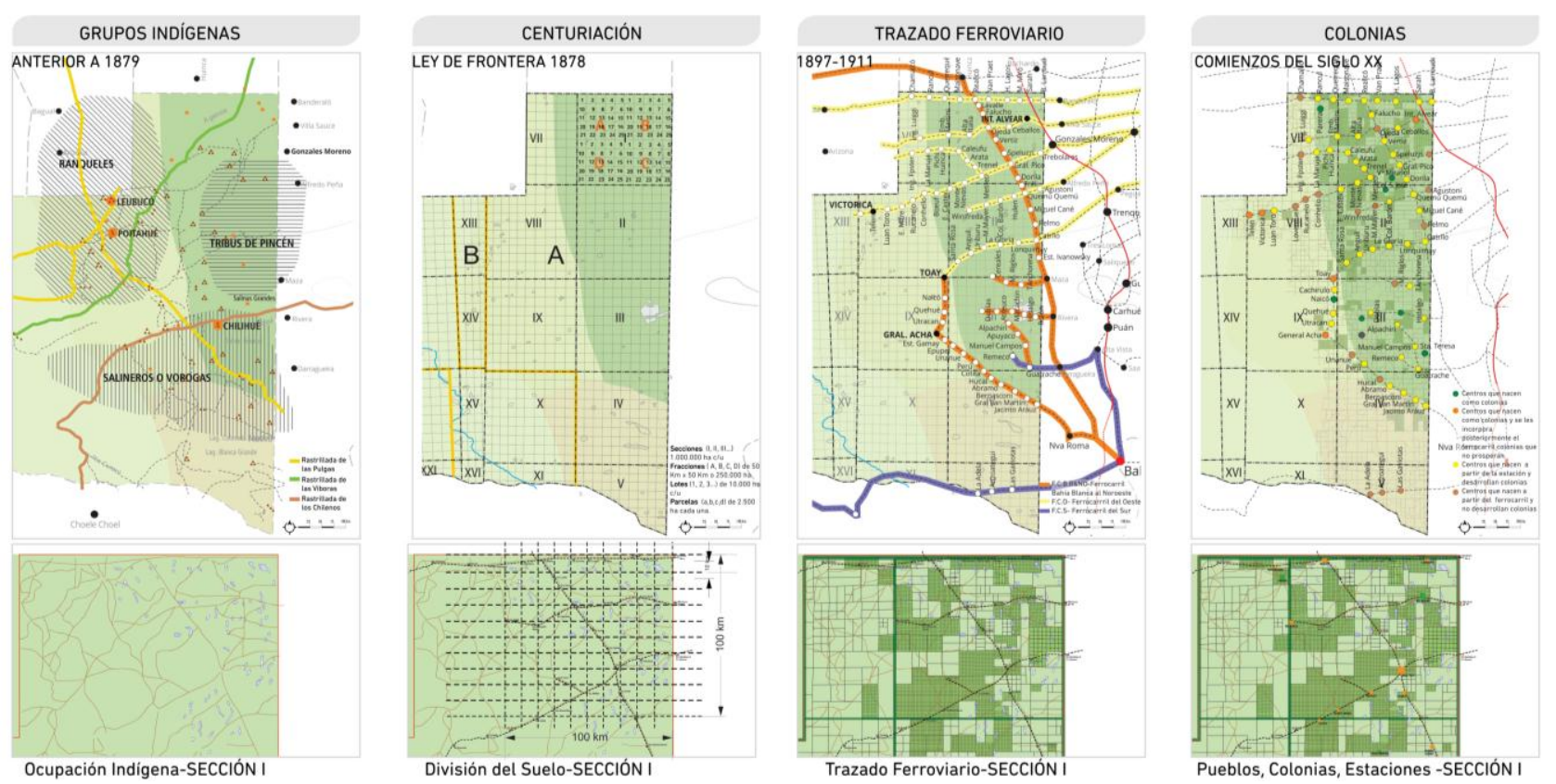

Gráfico 4. Elaboración propia de la ocupación indígena (rastrilladas), centuriación del suelo, colonización, trazado ferroviario y trazado de los pueblos (Martínez, 2016).

desde Trenque Lauquen (Buenos Aires) hasta Santa Rosa de Toay y Toay, llegando casi conjuntamente con el F.C.B.B.\&NO (1898) y cerrando el circuito Bahía Blanca - Buenos Aires por Toay. Por el Sureste, en 1899, ingresa la última compañía y la que menor desarrollo de rieles presenta en La Pampa, el Ferrocarril del Sur -F.C.S-, con Directorio en Londres, desde el Puerto de Bahía Blanca hasta Neuquén, atravesando el límite SE del territorio de La Pampa. En 1884, a poco tiempo de culminada la Expedición al Desierto, el suelo que hoy ocupa la Provincia de La Pampa es declarado Territorio de Gobernación Nacional. 
Las actividades económicas ligadas a la producción agrícola rural, como los procesos de producción, acopio, comercialización y distribución, determinan la permanente convivencia del paisaje urbano y rural. Tanto las edificaciones e infraestructuras que integran el cuadro de la estación y las que se desarrollan en los campos, conviven con el paisaje de La Pampa (Ramos, 1991; Bulgheroni, 1994).

En cada chacra se dispone de la tecnología que permite la puesta en producción agrícola y ganadera extensiva: molinos de viento, tanques australianos, maquinarias agrícolas, galpones de chapa como depósitos. Desde los caminos se percibe la producción de las chacras, el color y textura de los campos, las tranqueras, las viviendas rurales con galerías de techos de chapas y perfiles de acero, traídos junto al material de las estaciones, en barcos desde Inglaterra.

En corto plazo, se produce en La Pampa un importante proceso sincrético de absorción de tecnologías materiales y técnicas-. El ingreso ferroviario incorpora elementos culturales y tecnológicos europeos británicos principalmente- con la instalación de nuevos artefactos y desarrollo técnico. Mientras el trazado de las colonias, supone la incorporación cultural de miles de agricultores europeos, por otro lado comienza un período de dominación económica y tecnológica, bajo la bandera política de la civilización, proceso en que las preexistencias de la ocupación indígenas se debilitan y dan comienzo a un proceso de transculturización. (Ramos, 1991).

\subsection{Los elementos de la construcción de los pueblos}

La conjunción del trazado rural de las colonias y el trazado del ferrocarril determina la localización de los pueblos a partir de los cuadros de las estaciones, dando origen al patrón de asentamientos urbano-territorial dominante de La Pampa. ${ }^{9}$ Entre los elementos principales de la configuración física y paisajística de los centros urbanos y/o rurales, se encuentran el trazado ferroviario del cuadro de la estación y su determinación en el trazado urbano, las calles principales, las plazas y los edificios públicos singulares. ${ }^{10}$

El ferrocarril avanza sobre el territorio ocupándolo, disponiendo los cuadros de la estación y demás dependencias en el trazado urbano necesarias para su desarrollo. En el Territorio Nacional de La Pampa el $84 \%$ de la totalidad de los pueblos se trazan a partir del ferrocarril, por lo que el cuadro de la estación constituye el espacio más significativo y representativo, el verdadero germen inicial del proceso urbanizador, integrando regionalmente los pueblos entre sí y con los puertos de Buenos Aires y Bahía Blanca, y a través de estos, al mundo. ${ }^{11}$

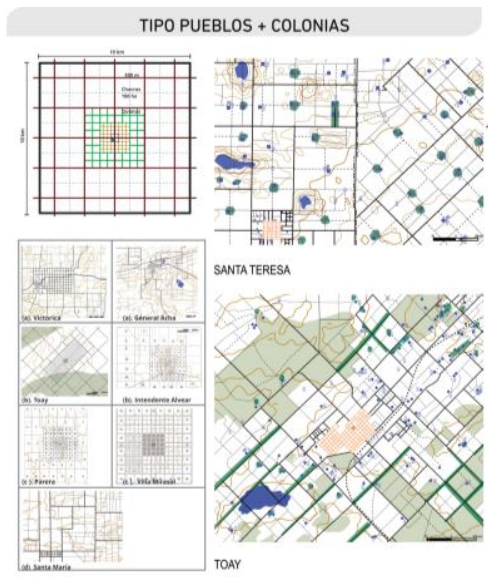

Gráfico 5. Elaboración propia patrones:
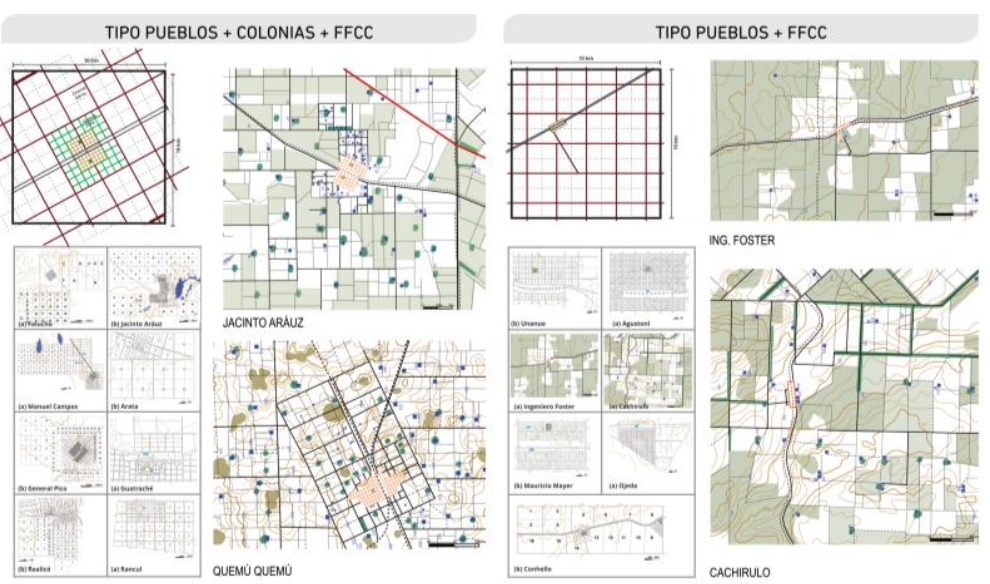

(Martínez, 2016)

\footnotetext{
${ }^{9}$ Tres patrones caracterizan el procesos de ocupación y urbanización pampeano: el patrón que integra pueblos y colonias, el patrón que integra pueblos+ ferrocarril, el patrón que integra pueblos+ colonias+ ferrocarril. Éste último es patón dominante y más exitoso del desarrollo pampeano. (Martínez 2016)

${ }^{10}$ En esta investigación, se establece una posible clasificación de la condición de desarrollo de los pueblos, en base a la cantidad de población que los mismos presentan para 1935, según datos disponibles del Censo Nacional de este año: a) Centros urbanos: la población inicial supera los 500 habitantes (según Censo Territorio Nacional de La Pampa 1935). Corresponde al 58\% de los pueblos analizados; b) Centros de servicios rurales: la población inicial no supera los 500 habitantes (según Censo Territorio Nacional de La Pampa 1935). Corresponde al $42 \%$ de los pueblos analizados.

${ }^{11} 97$ estaciones, 79 pueblos, de los cuales 66 son trazados urbanos ferroviarios y se anexan estaciones a 7 centros trazados en base a colonias militares y/o agrícolas. Cerca de un $80 \%$ de los pueblos de La Pampa presenta estaciones ferroviarias.
} 
Cada compañía, de las tres que entran a La Pampa, presenta una tipología de cuadro de estación y edificaciones singulares, que identifican a cada empresa. El tamaño de los cuadros de estación y la disposición del edificio de pasajeros, es uno de los rasgos más importantes de la configuración resultante de los pueblos. Su trazado y dimensiones definen las direcciones en que se organiza la trama urbana, la distribución de las manzanas a uno y otro lado, sus dimensiones y el tamaño de las calles.

Las proporciones de cada cuadro dependen de la compañía ferroviaria como también la disposición y localización de la estación de pasajeros -simétrica o no- dentro del cuadro. De este modo, la dirección que adopta el trazado urbano está condicionada a la disposición de los cuadros de estación, con dominancia Norte-Sur, cuando el ferrocarril conecta con el Puerto de Bahía Blanca; y Este-Oeste, cuando lo hace con el Puerto de Buenos Aires. A uno y otro lado del cuadro de estación, se disponen manzanas en hileras cuya cantidad y proporciones determinan el módulo del tamaño de este. En la mayoría de los pueblos ferroviarios se desarrollan hileras de 8 manzanas paralelas al cuadro de estación; el número de hileras varía a uno y otro lado de la vía, pudiendo ser 3, 5, 7, 9. La superficie trazada depende del rol económico principal que el pueblo desarrolle.

En el cuadro de la estación se dispone toda la infraestructura necesaria para abastecer las locomotoras, tanques de agua en torres de acero o mampostería acompañados de molinos de viento, talleres de reparación de máquinas, viviendas de obreros y jefes del ferrocarril. La estación de cargas y pasajeros constituyen elementos referenciales que se detallan en el análisis de la escala urbana. El corredor ferroviario concentra funciones industriales (depósito y venta de granos), comerciales y de servicios en sus bordes (fondas, hoteles, almacenes, venta de inmuebles, entre otros). La tipología de la estación se define en base a la localización de ésta, sus funciones y dependencias a fines.

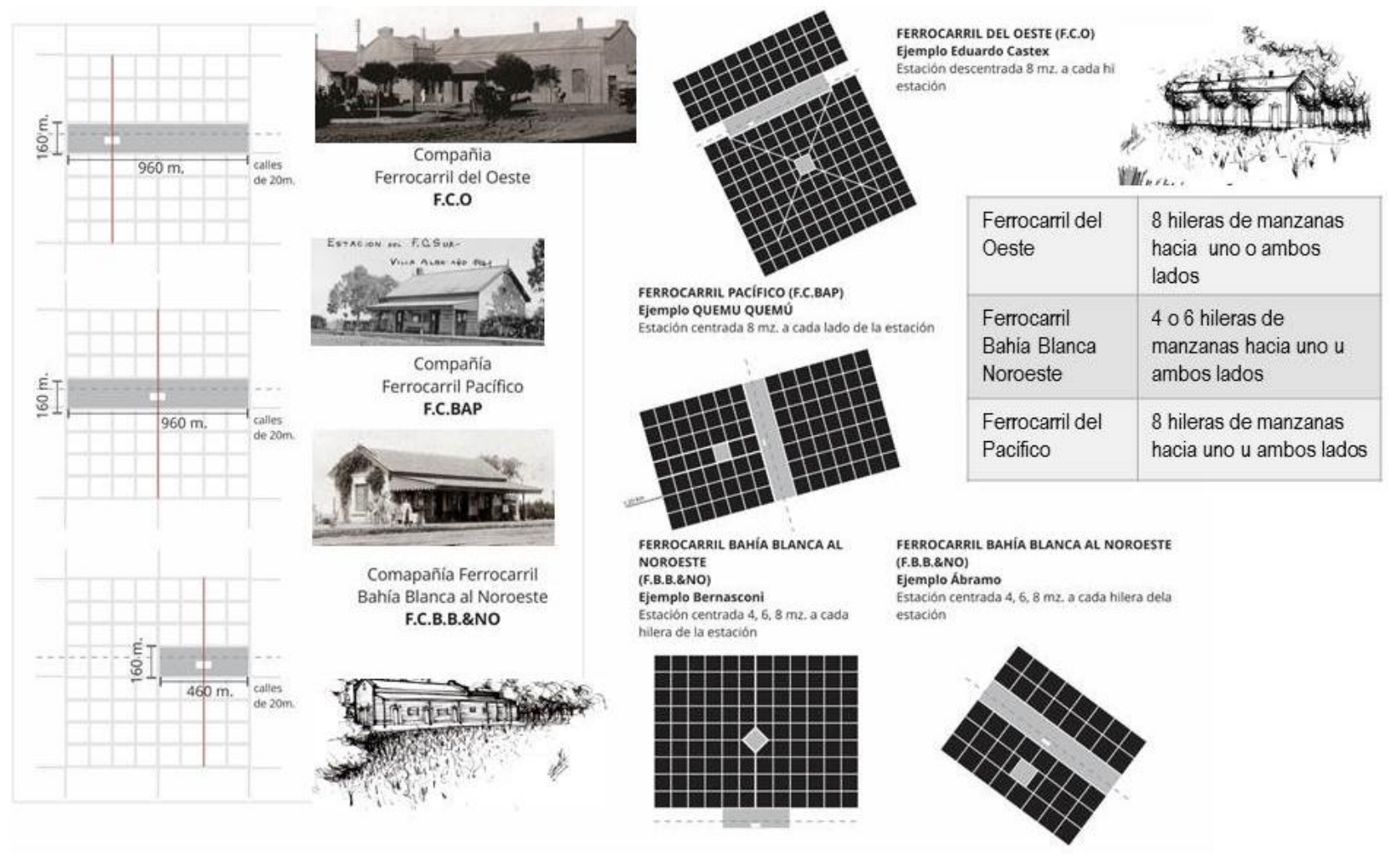

Gráfico 6. Elaboración propia tipos de trazados a partir del cuadro de la estación y la compañía ferroviaria (Martínez, 2016)

En torno al distrito ferroviario se disponen manzanas de tamaño promedio $100 \times 100 \mathrm{~m}$, las que se alinean paralelas a éste, conforme la modulación base adoptada. En cada polígono cuadrado de 25 ha. de superficie se disponen 16 manzanas. Hay excepciones en el número y formas de las manzanas, adoptando formas rectangulares en algunos casos, en vez de cuadrículas perfectas. No existe un criterio homogéneo en la subdivisión parcelaria de las manzanas ya que la cantidad de lotes va in crescendo según el proceso especulativo que se desarrolla sobre el suelo.

El ejido integra el trazado urbano del pueblo y el trazado rural de las colonias, en superficies variables entre 
8.000 y 10.000 ha. El área urbana presenta un modesto trazado de manzanas, un área de quintas (entre 5 y 20 ha. c/u) y un área de chacas (entre 45 a 200 ha. c/u) en sendos perímetros. En algunos pueblos, el área de quintas es reducida y se desarrollan mayormente chacras de grandes dimensiones.

En los pueblos ferroviarios, la rotación de la trama urbana conforme la dirección dominante de las ferrovías constituye una característica de estos. En general el trazado urbano y el trazado ferroviario adoptan la misma dirección, distinta a la dirección de la subdivisión del suelo agrario que copia la dirección de la grilla territorial inicial. 12

En los pueblos, la actividad principal está ligada al desarrollo agrícola de las colonias, y los servicios derivados de estas: el acopio, comercialización y transporte de la producción de las colonias de su área de influencia. Las colonias siempre se encuentran vinculadas administrativamente a un centro, de donde se proveen de servicios y comunicación para la comercialización de los productos agrícolas (Gaignard, 1989).

Inicialmente, las vías carreteras de carácter regional más importantes son los caminos principales que corren paralelos al trazado ferroviario y abrazan el cuadro de la estación, dando acceso a un sistema de caminos rurales transversales que facilitan la concentración de la producción en el ferrocarril.

A escala urbana, además de las calles paralelas al cuadro, son importantes funcionalmente las perpendiculares con paso a nivel a ambos lados de estación y la arteria comercial. ${ }^{13} \mathrm{La}$ calle comercial se conforma entre dos polos: la plaza y la estación de pasajeros. Según su escala, el centro puede desarrollarse linealmente, extenderse en forma de área o no desarrollarse. En algunos centros, esta calle está diseñada como bulevar. ${ }^{14}$

Las plazas se desarrollan a uno y otro lado de la vía, según las características del trazado. Su disposición es variable, pudiendo o no estar alineadas respecto al edificio de la estación, a distancias de 1, 2 o 3 manzanas. La plaza puede localizarse en una manzana más o menos central del trazado en cuadrícula, o desplazada según la posición de la estación (y ésta en función de la compañía ferroviaria de que se trate). La forma, orientación y dimensiones que las plazas adoptan es variable, pudiendo estar rotadas respecto del trazado en cuadrícula o ser una manzana vacía del conjunto trazado.

Si bien los edificios públicos se localizan en torno a la plaza, en el caso de los pueblos de origen ferroviario, esto no sucede en el inicio sino que la incorporación se produce luego con el desarrollo del centro y la vida institucional. Provisoriamente, las actividades cívico-administrativas, como jueces de paz y policía, se localizan en edificios de uso transitorio; más tarde, cuando se consolida su uso, los principales equipamientos públicos, como municipalidad e iglesia, se localizan en edificios singulares.

En estos pueblos, el distrito que contiene la estación opera como centro en el trazado, conformando con la plaza y el eje comercial entre estas, tres subsistemas característicos: el cívico, el comercial y el ferroviario. A pesar de la aparente homogeneidad de la configuración inicial resultante de los trazados urbanos, existen sutiles diferencias según la dirección y forma del cuadro de la estación, la posición de la estación de pasajeros, la disposición de las plazas, y del trazado urbano a uno o dos lados del cuadro de la estación. ${ }^{15}$

\section{REFLEXIONES}

El caso de la urbanización del territorio de La Pampa, constituye un caso de urbanización privada de una extensión nunca antes pensada. Entre 1882 y 1928, se trazan en La Pampa $80.000 \mathrm{Km}^{2}$ de tierras, 15.000 $\mathrm{Km}^{2}$ de colonias, cerca de 100 estaciones y 79 pueblos, $1500 \mathrm{~km}$ de vías con conexión a dos puertos. La

\footnotetext{
${ }^{12}$ Según como se articulan las direcciones del trazado urbano, el trazado rural y el ferrocarril, con la grilla territorial regular de la primera división del territorio, se dan diferentes situaciones: a) El trazado urbano, la subdivisión del suelo agrario y el trazado ferroviario mantienen la misma dirección de este último, independiente de la grilla territorial inicial; b) El trazado urbano y el trazado ferroviario adoptan la misma dirección, distinta a la dirección de la subdivisión del suelo agrario que copia la dirección de la grilla territorial inicial; c) El trazado urbano y la subdivisión del suelo agrario mantienen la misma dirección, distinta a la dirección de la línea ferroviaria.

${ }^{13}$ Calles con paso a nivel y calles de borde al cuadro de la estación - limítrofes a los bordes del distrito del ferrocarril- se extienden hacia las rutas o caminos regionales.

${ }^{14}$ Calles como boulevard o avenidas. Representan ejes perpendiculares al cuadro de la estación que se proyectan hacia uno $u$ ambos lados de la vía. Se distinguen por su diseño y por contener las plazas principales; por lo general, su caracterización es más paisajística que funcional.

${ }^{15}$ Algunos elementos como las diagonales, presentes en el trazado de La Plata, son adoptadas por algunos pueblos ferroviarios tales como Ingeniero Luiggi, Eduardo Castex.
} 
verdadera fiebre del proceso urbanizador se produce entre 1901 y 1911, período en el cuál se trazan en La Pampa más de 50 pueblos.

Para 1935, los pueblos trazados bajo el patrón que engloba pueblos+colonias+ferrocarril presentan ventajas comparativas por su localización, poblamiento, disponibilidad de ferrocarril y desarrollo prospero de colonias. Por su localización: están localizados en las mejores tierras correspondientes a la sub zona del valle pampeano; por su disponibilidad de ferrocarril: están integrados regional e internacionalmente y con los puertos, mediante 3 compañías ferroviarias; por su desarrollo prospero de colonias: todos están colonizados para 1930, haciendo de La Pampa uno de los principales productores del país.

De todos, es el tipo ferroviario el que mejor se adapta a los objetivos de producción y desarrollo económico de La Pampa en el mercado mundial, una red de ferrovías en abanico estructura este territorio desde los puertos de Bahía Blanca y Buenos Aires, y define un sistema de nodos o trazados urbanos que integran el espacio productivo circundante mediante el desarrollo de las colonias. Un modelo pensado para sacar la producción e ingresar productos elaborados. Todo ello ideado en un modelo de integración cultural, tecnológica con el mundo desarrollado, acercando la civilización al campo, dejando atrás la barbarie.

En la caracterización de los elementos de la construcción del territorio y de los pueblos es posible demostrar como los elementos del tipo característico dominante que integra en un mismo patrón pueblos+ferrocarril+ colonias están presentes en dos escalas: territorial y urbana. El proyecto del territorio es el proyecto de los pueblos, y viceversa. A partir de esta dialéctica, es posible inferir ciertos rasgos comunes, que constituyen las características principales que identifican el paisaje urbano- territorial pampeano.

El protagonismo de la naturaleza frente a la escala inconmensurable del espacio infinito, la construcción de referencias, limites, espacios significativos. El soporte natural, el paisaje resultante, con tenues diferencias caracteriza fuertemente los pueblos de La Pampa. La presencia de un accidente menor - sobre la planicieidentifica el espacio, lo referencia. En La Pampa el espacio -el susurro permanente del viento, la arena en movimiento, el olor a pastizales, el color de los campos- es el protagonista. La vista es el horizonte, siempre presente en la finitud de la calles, de los caminos, y del entorno fuera de las manzanas urbanas.

La geometría como recurso potente de ocupación y transformación del territorio. El macro trazado en cuadrícula arma una red invisible primero, luego perimetrada por hilos de alambre, más tarde sendas amplias de tierra, finalmente enfilan casas en sus bordes. La ocupación pampeana es el tablero de un juego de ajedrez, donde algunos casilleros se ocupan, en tanto otros que se ocuparon ahora están vacíos.

Las sutiles diferencias frente a la aparente homogeneidad del paisaje urbano- territorial. Tres patrones de asentamiento típicos pampeanos se conjugan en tres grandes regiones definiendo áreas homogéneas con características diferenciables. Aún en el patrón dominante de los pueblos ferroviarios, las distintas compañías ferroviarias determinan variantes en los elementos de la configuración de los trazados urbanos: manzanas, plazas, bulevares. Los pueblos de La Pampa se diseñan e integran a una estrategia de conjunto que - más allá de criterios repetitivos- representan características de los pueblos pampeanos.

El desarrollo infraestructural ferroviario y su importancia en la construcción morfológica del territorio y de los pueblos. El ferrocarril rota la ortogonalidad de la grilla con la que se fracciona el suelo, definiendo la localización de los pueblos, la dirección de su trama, la disposición de las manzanas, las quintas, las chacras en su entorno. El ferrocarril es esencialmente acceso, es camino, es integración en todas las escalas. La red indígena de antiguas rastrilladas desdibujadas sobre el piso del desierto, comienza a vibrar nuevamente en La Pampa. Un sistema de rieles se teje omnipresente, llevando por las venas del mundo, materia prima y productos, desde y hacia corazón de La Pampa.

La dialéctica entre los elementos de construcción del espacio urbano y rural. El campo penetra en la ciudad, una y mil veces, el campo es ciudad, la ciudad es campo. La ocupación pampeana es el tablero de un juego de ajedrez, donde algunos casilleros se ocupan, en tanto otros que se ocuparon ahora están vacíos.

Mediante este estudio se señala la permanencia de los elementos de construcción del territorio en el hecho urbano, demostrando como la ocupación comienza en la escala regional. Por primera vez en la historia argentina, tan amplio territorio, que excede el meridiano $\mathrm{V},{ }^{16}$ es puesto en producción, colonizado, urbanizado con una lógica constante, un serie de reglas establecidas por la voluntad política, fundamentadas en las ideologías dominantes, materializada con los conocimientos técnicos de agrimensores independientes, y

\footnotetext{
${ }^{16}$ Límite oriental que separa la provincia de Buenos Aires con el entonces Territorio Nacional de La Pampa.
} 
llevada adelante por la voluntad de los propietarios de los campos.

Los elementos analizados constituyen en tanto rasgos fundamentales de la identidad pampeana, insumos para la proyectarían del territorio y sus pueblos. La construcción de los pueblos de La Pampa, es la construcción de un proyecto territorial. Los elementos de construcción del territorio están presentes en los pueblos determinando, posibilitando y condicionando el hecho urbano.

\section{BIBLIOGRAFIA}

AVELLANEDA, Nicolás (1865) Estudios sobre las Leyes de tierras públicas. Buenos Aires.

(1876) Ley Nacional $N^{\circ} 857$ de Inmigración y Colonización o Ley Avellaneda.

BULGHERONI, Raúl. (1994) Argentina imagen de un país. Buenos Aires: Summa Pampeana. p. 211.

CANO, Eduardo (coord.) (2004) Inventario integrado de los recursos naturales de la provincia de La Pampa: clima, geomorfología, suelo y vegetación. INTA. Universidad Nacional de La Pampa. Buenos Aires.

CAVIGLIA, Jorge; LORDA, Héctor y Jorge LEMES (2010) Caracterización de las unidades de producción agropecuarias en la provincia de La Pampa. Ediciones INTA. La Pampa

FERRARI, Mónica (2010): Patrimonio Ferroviario en el Noroeste Argentino La Línea Jujuy- La Quiaca. Tesis de doctorado. Universidad Pablo de Olavide de Sevilla. Departamento de Geografía, Historia y Filosofía. España.

FOGLIA, Ma. Elena y Noemí GOYTIA (1991) El patrimonio urbanístico ambiental de las regiones argentinas: los poblados históricos del norte cordobés. Secretaría de Turismo de la provincia de Córdoba.

(1991) Rehabilitación de poblados históricos del Norte Cordobés: el caso de Tulumba. Cuaderno de la Maestría en Desarrollo Urbano. Universidad Nacional de Córdoba., FAUDI. Córdoba.

FOGLIA, Ma. Elena (comp.) (1995) Cultura y territorio: los procesos de urbanización latinoamericanos y argentinos. Documento de la Maestría en Desarrollo Urbano y Regional. Universidad Nacional de Córdoba, FAUDI. Córdoba.

FOGLIA, Ma. Elena. (1998) Bases para una historia urbana de la Provincia de Córdoba. Reflexiones sobre los procesos históricos de ocupación del territorio. Teorías y Modelos. Argentina y provincia de Córdoba. Maestría en Desarrollo Urbano. Cuadernos de Estudio. Universidad Nacional de Córdoba, FAUDI. Córdoba.

(2000) El sistema urbano y la configuración de la ciudad. Documento de la Cátedra de Urbanismo de la Universidad Nacional de Córdoba, FAUDI. Córdoba.

(2001) Bases para una historia urbana de Córdoba. Informe SECyT. Universidad Nacional de Córdoba, FAUDI. Córdoba.

FOGLIA, Ma. Elena et al. (2003) "Bases para una historia urbana, Provincia de Córdoba. Argentina". En Cuadernos de investigación urbanística CIUR $N^{\circ} 37$. Madrid.

GAIGNARD, Romain. (1989) La Pampa Argentina. Ocupación, poblamiento, explotación: de la Conquista a la Crisis Mundial (1910-1930). Soler. Buenos Aires.

GOYTIA, Noemí (1995) "La identidad en relación a la escala". En Curso Taller Rehabilitación y Desarrollo de poblados históricos. Maestría en Desarrollo Urbano y Regional. UNC, FAUDI. Córdoba.

GUTIERREZ, Ramón (1976) "La política fundacional y la ampliación de fronteras". En Revista 2cmConstrucción de la ciudad $N^{\circ} 19$. Barcelona. pp. 10-21.

LLUP, Andrea y SALOMON TARQUINI, Claudia (compiladoras). Historia de La Pampa, Argentina. Sociedad, política, economía. Desde los poblamientos iniciales hacia la provincionalización. UNLPam. Santa Rosa, 2008.

MARTINEZ, Mónica (2016) Los pueblos del desierto: el proceso de ocupación y urbanización del Territorio Nacional de La Pampa, Argentina. Tesis de doctorado. Director: Dra Arq. Joaquín Sabaté; Codirectora: Dra Arq. Mónica Ferrari. Universidad Politécnica de Cataluña. España.

MOLLO, Norberto y Carlos DELLA MATTIA. (2009) "Rastrilladas y parajes de Mamúl Mapu". En MARTINI, Yoli; PEREZ ZAVALA, Graciana y Yanina AGUILAR (compiladoras). Las sociedades de los paisajes áridos y semiáridos del centro Oeste argentino. Universidad Nacional de Río Cuarto.

PÉREZ AMUCHASTEGUI, Antonio (1969) “Los Gringos”. En GIBELLI, Nicolás (director responsable). Crónica Histórica Argentina. Tomo IV. Editorial Codex. Buenos Aires.

RAMOS, Jorge (1991) La aventura de la pampa argentina: Arquitectura. Ambiente y Cultura. Corregidor. Buenos Aires.

RANDLE, Patricio (1969) La ciudad pampeana. Editorial Eudeba. Buenos Aires

SABATÉ, Joaquín (1996) Forma del territorio y proyecto. Universidad Politécnica de Cataluña. Barcelona. (1998) Elementos para la construcción de un proyecto territorial: programa de las lecciones. 
Universidad Politécnica de Cataluña. Barcelona.

(1999) Las reglas de la forma urbana: programa de las lecciones. Universidad Politécnica de Cataluña. Barcelona.

(2004) Patrimonio y Proyecto Territorial. Colonias, Sèquia de Manresa y Delta del Llobregat. Diputación de Barcelona.

(2004) "Elementos para la construcción de un proyecto territorial". En SABATÉ, Joaquim (coordinador), Patrimonio y proyecto territorial. Colonia, Séquia de Manresa y Delta del Llobregat. Diputación de Barcelona.

Cataluña. Barcelona. (2005) $E$ 